\title{
A rare case of kissing gastric ulcers caused by trauma
}

\author{
Yan Zhang ${ }^{1}$, Yafei Zhang ${ }^{2}$, Shiyang $\mathrm{Ma}^{3, *}$, Haitao $\mathrm{Shi}^{3}$, Xiaolan $\mathrm{Lu}^{3}$ \\ ${ }^{1}$ Department of Cardiology, Weinan Central Hospital, Weinan, Shaanxi, China; \\ ${ }^{2}$ Department of General Surgery, Second Affiliated Hospital, School of Medicine, Xi'an Jiaotong University, Xi'an, Shaanxi, China; \\ ${ }^{3}$ Department of Gastroenterology, Second Affiliated Hospital, School of Medicine, Xi'an Jiaotong University, Xi'an, Shaanxi, China.
}

\begin{abstract}
Summary We present a rare case of a 32-year-old female with gastric ulcers caused by pressure from trauma. The patient was diagnosed with stress-related acute gastritis by a local hospital and she was discharged 1 week later after her symptoms improved. She was given oral proton pump inhibitors (PPI). However, epigastric pain intensified, so the woman was seen at this Hospital 3 days later. Gastric endoscopy revealed kissing ulcers in the lower body of the stomach. A point worth mentioning is that the kissing gastric ulcers were caused by trauma due to impact with the steering wheel.
\end{abstract}

Keywords: Kissing gastric ulcers, pressure ulcer, trauma

\section{Introduction}

A pressure ulcer is a lesion in the skin and/or underlying tissue, usually over bony prominences, caused by pressure and/or shear (1). Pressure is not a common cause of peptic ulcers. Pressure ulcers in the stomach are rare, as evident in the fact that there are only 4 reported cases of those ulcers (2-5). The causes and mechanisms of pressure ulcers differ; some are due to the use of non-steroidal anti-inflammatory drugs (NSAIDs) and some are due to trauma. In the case reported here, kissing gastric ulcers developed after blunt abdominal trauma.

\section{Case report}

A 32-year-old woman abruptly applied the brakes while driving and impacted the steering wheel, and she was brought to the Emergency Department of a local hospital for abdominal pain. The woman had no history of alcohol or drug abuse, was not using NASIDs, and she was negative for a Helicobacter pylori infection. Right after the accident, the woman had severe and

Released online in J-STAGE as advance publication September 12, 2016.

*Address correspondence to:

Dr. Shiyang Ma, Department of Gastroenterology, Second Affiliated Hospital, School of Medicine, Xi'an Jiaotong University, 157 West 5th Road, Xi'an 710004, China.

E-mail: shiyangma@163.com persistent epigastric pain radiating to her back, and this pain was alleviated by bending at the waist. The woman indicated that she had nausea and vomiting but no fever or diarrhea. Her vital signs were stable and a physical examination indicated marked tenderness with focalized guarding in the epigastric region. Abdominal computed tomography (CT) was normal and laboratory results were all within normal limits.

The woman was diagnosed with stress-related acute gastritis by the hospital and she was discharged 1 week later after her symptoms improved. She was given oral proton pump inhibitors (PPI). However, epigastric pain intensified, and the woman was seen at this Hospital 3 days later. Gastric endoscopy revealed kissing ulcers in the lower body of the stomach (Figure 1). Endoscopic ultrasound (EUS) was performed and the ulcers had penetrated into the deep mucosal layer (Figure 2). An easily digestible diet was recommended and oral PPI were continued for 6-8 weeks. The patient had no further complaints of abdominal pain a month later during follow-up. Biopsy samples obtained from the margin and base of the ulcers ruled out an H. pylori infection.

\section{Discussion}

Peptic ulcers are highly prevalent among patients in Gastroenterology. The most common causes of gastric ulcers are an H. pylori infection and use of aspirin or non-steroidal anti-inflammatory drugs. Other causes include acute pancreatitis, smoking, acute stress, congenital anomalies, a gastric motility disorder, 


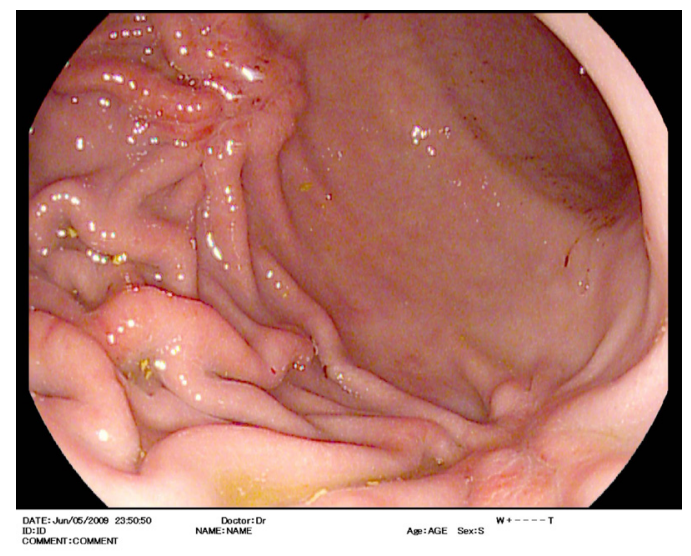

Figure 1. An endoscopic examination revealed kissing ulcers in the body of the stomach.

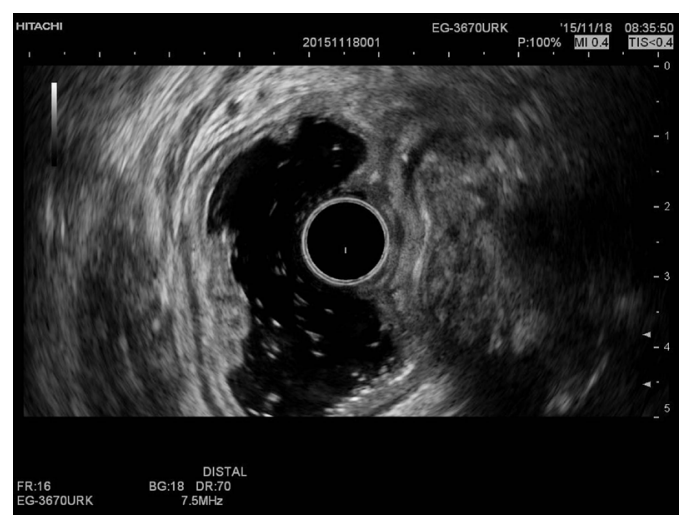

Figure 2. EUS revealed that the ulcer had invaded submucosa layer.

foreign bodies, and trauma. In the current case, ulcers were caused by trauma.

Given the abrupt development of the gastric ulcers, common causes were ruled out. Research has shown that stress causes peptic ulcers much like those associated with $H$. pylori infection or non-steroidal anti-inflammatory drugs (6). Endoscopic studies have revealed that between $74-100 \%$ of critically ill patients have stress-related mucosal damage within $24 \mathrm{~h}$ of admission (7). Although multiple factors were presumably responsible for causing gastric ulcers in the current patient, stress was not one such factor.

In terms of morphology, peptic ulcers are more common in the anterior wall of the duodenal bulb, gastric angle, and gastric antrum. Kissing gastric ulcers in both the anterior and posterior gastric wall might be the result of acute gastric ischemia caused by external force. A reduction in mucosal blood flow resulted in the sequence of events that led to formation of an acute gastric mucosal lesion. To the extent known, mucosal damage associated with acute shear is also a cause of gastric ulcers. When the patient is in a stationary state, shear may occur between the fixed skin and the moving subcutaneous tissue, and the results of this shear may be more evident near the bony prominences $(8-10)$. This may explain why the ulcers were in front of the spine in the current patient. A series of changes will occur after shear damage, such as neuroendocrine changes and activation of immune cells. Pathological biopsy will reveal accumulation of a large number of inflammatory cells such as neutrophils, monocytes/macrophages, and lymphocytes in the microcirculation, and inflammatory mediators that are subsequently released will aggravate the injury to gastric mucosa (l).

In the current case, the patient was fasting on the day of the accident. Therefore, a bold conjecture may be advanced: food in the stomach may be protective since it may absorb or buffer pressure to some extent. An appropriate treatment for peptic ulcers may be fasting with parenteral nutrition, use of PPI, use drugs that protect the mucosa, or even gastrointestinal decompression.

\section{References}

1. Oliveira Costa AC, Sabino Pinho CP, Almeida dos Santos AD, Santos do Nascimento AC. Pressure ulcer: Incidence and demographic, clinical and nutrition factors associated in intensive care unit patients. Nutr Hosp. 2015; 32:2242-2252. (in Spanish)

2. Abdukadyrov MA, Baizakov UB. A rare case of a silent, "kissing" or "mirror(-image)" ulcer of the stomach. Sov Zdravookhr Kirg. 1966; 2:61-62. (in Russian)

3. Dedic M. "Kissing ulcers" of the stomach. Srp Arh Celok Lek. 1963; 91:79-85.

4. Mensier A, Bounoua F, Beretvas G, Mosoi A, Dardenne S. Kissing gastric ulcers causing acute pancreatitis and portal biliopathy: What's the link? JOP 2013; 14:646648.

5. Verhaeghe M, Depadt G, Laurent JC, Clay A. Transformed double gastric ulcer (kissing ulcer). Gastrectomy. 12-year recovery. Lille Med 1973; 18:691695. (in French)

6. Levenstein S, Rosenstock S, Jacobsen RK, Jorgensen T. Psychological stress increases risk for peptic ulcer, regardless of Helicobacter pylori infection or use of nonsteroidal anti-inflammatory drugs. Clin Gastroenterol Hepatol. 2015; 13:498-506.e1.

7. Mutlu GM, Mutlu EA, Factor P. GI complications in patients receiving mechanical ventilation. Chest. 2001; 119:1222-1241.

8. Correa GI, Fuentes M, Gonzalez X, Cumsille F, Pineros JL, Finkelstein J. Predictive factors for pressure ulcers in the ambulatory stage of spinal cord injury patients. Spinal Cord. 2006; 44:734-739.

9. Baumgarten M, Margolis DJ, Localio AR, Kagan SH, Lowe RA, Kinosian B, Abbuhl SB, Kavesh W, Holmes $\mathrm{JH}$, Ruffin A, Mehari T. Extrinsic risk factors for pressure ulcers early in the hospital stay: A nested case-control study. J Gerontol A Biol Sci Med Sci. 2008; 63:408-413.

10. Keryln C. Wound care manual. 5th Edition, Western Australia, Osbome Park: Silver Chain Foundation. 2005; 82-93.

(Received July 28, 2016; Revised August 19, 2016 ; Accepted August 31, 2016) 